\title{
NORMAL FORMS FOR LIE ALGEBROIDS
}

\author{
JEAN-PAUL DUFOUR \\ Mathématiques, Université Montpellier II \\ Pl. E. Bataillon, 34095 Montpellier Cedex, France \\ e-mail:dufourj@math.univ-montp2.fr
}

\begin{abstract}
In this paper we will, first, give a local splitting theorem for Lie algebroids which shows that we have a splitting in the product of a tangent bundle and a rank 0 Lie algebroid. We give a very simple way to prove the unicity of the transversal structures. We also give a linearization theorem for these rank 0 algebroids. All these results are part of the "folklore" of the domain and are not very difficult, but, to my present knowledge, they are unavailable in the literature.
\end{abstract}

1. Splitting theorem. A Lie algebroid is a vector fiber bundle A, fibering over the base manifold $M$, equipped with two extra structures:

a- a Lie bracket structure [, ] on the space $\Gamma A$ of sections of $A$ b- a $C^{\infty}(M)$-linear mapping $\sharp: \Gamma A \rightarrow \mathcal{X}(M)$, which is called the anchor of the Lie algebroid,

which satisfy the following two properties:

c- $\sharp$ is a Lie algebra homomorphism $(\sharp[\alpha, \beta]=[\sharp \alpha, \sharp \beta]$ for every sections $\alpha$ and $\beta)$, d- $[\alpha, f \beta]=\sharp \alpha(f) \beta+f[\alpha, \beta]$ for every sections $\alpha$ and $\beta$ and $f \in C^{\infty}(M)$.

This notion plays an important role in domains such that foliations (with or without singularities), Poisson manifolds, super-manifolds... (see, for example, [CW], [M], [W2]).

THEOREM 1. Let $A$ be a Lie algebroid on the base manifold $M$ with anchor $\sharp$ and section bracket [, ]. If the rank of Im $\sharp$ at the point $m$ of $M$ is $q$, then there is a trivialization of $A$, on a neighborhood of $m$ with local coordinates $\left(x^{1}, \ldots, x^{q}, y^{1}, \ldots, y^{s}\right)$, and a basis of sections $\left(\alpha^{1}, \ldots, \alpha^{q}, \beta^{1}, \ldots, \beta^{r}\right)$ such that $\sharp \alpha^{i}=\frac{\partial}{\partial x^{i}}$ and $\sharp \beta^{j}$ are independent of the $x^{k}$ for every $i, k=1, \ldots, q$ and $j=1, \ldots, r$. Moreover we have the relations

$$
\left[\alpha^{i}, \alpha^{j}\right]=\left[\alpha^{i}, \beta^{u}\right]=0
$$

for every $i, j=1, \ldots, q$ and $u=1, \ldots, r$ and

2000 Mathematics Subject Classification: Primary 53D17.

The paper is in final form and no version of it will be published elsewhere. 


$$
\left[\beta^{u}, \beta^{v}\right]=\sum_{w=1}^{r} \gamma_{w}^{u v} \beta^{w}
$$

for every $u, v=1, \ldots, r$, where the $\gamma_{w}^{u v}$ are functions which depend only on the coordinates $y^{1}, \ldots, y^{s}$.

Proof. To prove our result we will proceed by induction. There is nothing to prove if $q=0$, so we work with $q>0$. We suppose that there is an integer $k$ with $0 \leq$ $k<q$ satisfying the following condition: There is a trivialization of $A$, on a neighborhood of $m$ with local coordinates $\left(x^{1}, \ldots, x^{k}, y^{1}, \ldots, y^{s+q-k}\right)$, and a basis of sections $\left(\alpha^{1}, \ldots, \alpha^{k}, \beta^{1}, \ldots, \beta^{r+q-k}\right)$ such that

$$
\left[\alpha^{i}, \alpha^{j}\right]=\left[\alpha^{i}, \beta^{u}\right]=0, \quad \sharp \alpha^{i}=\frac{\partial}{\partial x^{i}}
$$

and $\sharp \beta^{u}$ are independent of the $x^{j}$, for every $i \leq k, j \leq k$ and $u=1, \ldots, r+q-k$.

Under this induction hypothesis, we have

$$
\left[\beta^{u}, \beta^{v}\right]=\sum_{i=1}^{k} a_{i}^{u v} \alpha^{i}+\sum_{w=1}^{r+q-k} \gamma_{w}^{u v} \beta^{w}
$$

where the $a_{i}^{u v}$ and the $\gamma_{w}^{u v}$ are some functions of, a priori, $x:=\left(x^{1}, \ldots, x^{k}\right)$ and $y:=$ $\left(y^{1}, \ldots, y^{s+q-k}\right)$. If we take the anchors of the two members of this equation, $\frac{\partial}{\partial x^{i}}$ doesn't appear in the first member but appears in the second member with coefficient $a_{i}^{u v}$; so we get that these coefficients all vanish. Moreover, Jacobi identity gives that $\left[\alpha^{j},\left[\beta^{u}, \beta^{v}\right]\right]$ vanishes, but this is also equal to $\sum_{w}\left(\frac{\partial}{\partial x^{j}} \gamma_{w}^{u v}\right) \beta^{w}$. Finally, up to a shrinking of the neighborhood of $m$ we consider, we can suppose

$$
\left[\beta^{u}, \beta^{v}\right]=\sum_{w=1}^{r+q-k} \gamma_{w}^{u v} \beta^{w}
$$

where the $\gamma_{w}^{u v}$ are functions of $y$ only.

As we have taken $k<q$, we can suppose, up to a reindexation, $\sharp \beta^{1}(m) \neq 0$. Then we can straighten this vector field: precisely, under a coordinate change $y \mapsto(t, z)(z$ belongs to $\mathbb{R}^{s+q-k-1}$; we don't touch $x$ ), we can suppose $\sharp \beta^{1}=\frac{\partial}{\partial t}$.

We add local sections of the form $a(t, z) \beta^{1}$ such that $\sharp \beta^{u}$, written in the coordinates $(t, z)$, doesn't have component in $\frac{\partial}{\partial t}$.

Now we will change the $\beta^{u}$, for $u>1$, in $\tilde{\beta}^{u}=\sum_{v=2}^{r+q-k} \theta_{v}^{u} \beta^{v}$ where $\Theta:=\left(\theta_{v}^{u}\right)$ is an invertible matrix with coefficients depending smoothly in $(t, z)$. We want to choose $\Theta$ such that $\left[\beta^{1}, \tilde{\beta}^{u}\right]=0$ for every $u>1$. This constraint reduces to the O.D.E.

$$
\frac{\partial \Theta}{\partial t}+\Theta \Gamma^{1}=0
$$

where $\Gamma^{1}$ is the matrix with coefficients $\gamma_{u}^{1 v}$, for $u>1$ and $v>1$. Up to a shrinking of the neighborhood of $m$ on which we work, we have good solutions to this O.D.E..

Remark that we have still $\left[\alpha^{i}, \tilde{\beta}^{u}\right]=0$ for every $u>1$ and $i \leq k$.

Now we can change notations and put $\alpha^{k+1}:=\beta^{1}, x^{k+1}:=t$, and we have replaced $k$ by $k+1$ in our starting hypothesis (the old $\beta^{u}$ for $u>1$ being replaced by $\tilde{\beta}^{u}$ and the old $y$ replaced by $z$ ). 
Starting with $k=0$, we can continue this procedure until $k=q$ and so, prove the theorem.

This theorem shows that $A$ decomposes locally as the direct product of a tangent algebroid and an algebroid with 0-rank at the origin. This "splitting theorem" improves the one presented in $[\mathrm{F}]$, though the proof is essentially the same.

A simple particular type of Lie algebroid is a bundle of Lie algebras: this is the case where every fiber is endowed with a Lie algebra structure, depending smoothly on the fiber; in that case the anchor is null.

COROLlary 2. If the distribution Im $\sharp$ has constant rank $q$ near $m \in M$, then the Lie algebroid $A$ is, over some neighborhood of $m$, the product of $T \mathbb{R}^{q}$ with a bundle of Lie algebras.

Proof. First use the splitting theorem. Then remark that the rank condition implies that the $\sharp \beta^{i}$ must vanish identically. This leads to the result.

In particular, if the rank at $m$ is maximal, i.e. equal to the dimension of the base, then $A$ is locally the product of a tangent algebroid and a (finite dimensional) Lie algebra.

2. Lie algebroid structure along the leaves. The distribution $\operatorname{Im} \sharp$ is involutive, and gives a foliation with singularities on the basis $M$. A leaf $F$ of the Lie algebroid $A$ is, by definition, a maximal integral submanifold of this distribution $([\mathrm{F}])$. Because, at any point of $F$, the anchor of any section is tangent to $F$ it makes sense to consider the Lie algebroid $\left.A\right|_{F}$, which is the restriction of $A$ to $F$.

Now we consider Ker $\left.\sharp\right|_{F}$ which is the fiber bundle over $F$ which has, at any point $m \in F$, the fiber $\operatorname{Ker}\left(\sharp_{m}: A_{m} \rightarrow T_{m} M\right.$ ) (for any fiber bundle $B$, we denote by $B_{x}$ its fiber at $x$ ). We can use the splitting theorem to show that this is a true fiber bundle, moreover it shows also that Ker\#| $\left.\right|_{F}$ is a bundle of Lie algebras where the Lie algebra structure of each fiber Ker $\left.\sharp\right|_{F m}$ can be defined by $\left[\alpha_{m}, \beta_{m}\right]_{m}=[\tilde{\alpha}, \tilde{\beta}](m)$ where $\tilde{\alpha}$ and $\tilde{\beta}$ are any sections with $\tilde{\alpha}(m)=\alpha_{m}$ and $\tilde{\beta}(m)=\beta_{m}$.

LEMMA 3. Up to isomorphism, the Lie algebra structure of each fiber of Ker $\left.\right|_{F}$ is independent of the fiber.

Proof. We work with the coordinates and the basis given by the splitting theorem. Then $F$ has the equation $y=0$ and Ker $\left.\sharp\right|_{F}$ has the local basis of sections $\left(\left.\beta^{1}\right|_{F}, \ldots,\left.\beta^{r}\right|_{F}\right)$ and the Lie algebra structure is, in every fiber, given by $\left[\beta^{u}(x), \beta^{v}(x)\right]=$ $\sum_{w=1}^{r} \gamma_{w}^{u v}(0) \beta^{w}(x)$. So the structure constants are independent of the fiber.

Proposition 4. We have the following exact sequence of Lie algebroids over F:

$$
\left.\left.0 \rightarrow \mathrm{Ker}\right|_{F} \rightarrow A\right|_{F} \rightarrow T F \rightarrow 0
$$

where the second mapping is the inclusion and the third mapping is given by $\sharp$.

This easy proposition gives a description of the Lie algebroid structure along the leaf $F$. In particular we see that $\left.A\right|_{F}$ is locally the product of $T F$ with a finite dimensional Lie algebra. There is an equivalence class of $(\operatorname{rank} A$-dim $F)$-dimensional Lie algebra attached to each leaf $F$. 
3. Transversal structure. Let $F$ be a leaf of our algebroid and $m$ a point of $F$. If $N$ is any transversal manifold to $F$ at $m$, we define $([\mathrm{F}])$ the transversal algebroid $A_{N}$ by the formula

$$
\left(A_{N}\right)_{m}=\left\{\alpha \in A_{m} ; \sharp \alpha \in T_{m} N\right\} .
$$

The anchor of $A_{N}$ is, by definition, the restriction of the one of $A$ and the bracket of sections $\alpha$ and $\beta$ being defined by $[\alpha, \beta]_{N}(m)=[\tilde{\alpha}, \tilde{\beta}](m)$ where $\tilde{\alpha}$ and $\tilde{\beta}$ are any extension of $\alpha$ and $\beta$ to a neighborhood of $m$. Using, for example, our local splitting, it is easy to show that this defines $A_{N}$ without ambiguity. If $N$ is exactly the manifold given by $x=0$ in our local splitting coordinates, then $A_{N}$ has the local trivialization given by the sections $\left.\beta^{1}\right|_{N}, \ldots,\left.\beta^{r}\right|_{N}$, the base $N$ admits local coordinates $y=\left(y^{1}, \ldots, y^{s}\right)$ and the "transversal" bracket is precisely given by

$$
\left[\left.\beta^{u}\right|_{N},\left.\beta^{v}\right|_{N}\right]=\left.\sum_{w=1}^{r} \gamma_{w}^{u v}(y) \beta^{w}\right|_{N} .
$$

Now remark that any transversal manifold $N$ to $F$ at $m$ has local equations $x^{i}=f^{i}(y)$ in the coordinates of the splitting theorem. We write $f(y):=\left(f^{1}(y), \ldots, f^{q}(y)\right)$. The following proposition gives a very elementary way to prove that $A_{N}$ is, up to an algebroid isomorphism, independent of the chosen transversal $N$ which passes to $m$.

Proposition 5. The smooth mapping

$$
\begin{gathered}
\Phi:=\sum_{i=1}^{q} a_{i} \alpha^{i}(x, y)+\sum_{u=1}^{r} b_{u} \beta^{u}(x, y) \mapsto \sum_{i=1}^{q}\left(a_{i}-\sum_{v=1}^{r} b_{v} \sharp \beta^{v}\left(f^{i}\right)(y)\right) \alpha^{i}(x-f(y), y)+ \\
\sum_{u=1}^{r} b_{u} \beta^{u}(x-f(y), y)
\end{gathered}
$$

defines a local isomorphism of the algebroid $A$ which sends the transversal $N$ to $x=0$.

Proof. By definition $\Phi$ is a vector bundle isomorphism over the local diffeomorphism $\phi:=(x, y) \mapsto(x-f(y), y)$. By construction, this $\phi$ maps $N$ to $x=0$. To show that $\Phi$ is an algebroid isomorphism we have first to prove the fromulae $\sharp\left(\Phi \circ \alpha \circ \phi^{-1}\right)=\phi_{*} \sharp \alpha$ for any section $\alpha$. It suffices to prove this for the sections of our basis. For $\alpha=\alpha^{i}$ we get

$$
\sharp\left(\Phi \circ \alpha \circ \phi^{-1}(x-f(y), y)\right)=\sharp \alpha^{i}(x-f(y), y)=\frac{\partial}{\partial x^{i}}(x-f(y), y)=\phi_{*} \sharp \alpha(x-f(y), y)
$$

as expected; for $\alpha=\beta^{u}$ we get

$$
\begin{gathered}
\left.\sharp\left(\Phi \circ \alpha \circ \phi^{-1}(x-f(y), y)\right)=\sharp \beta^{u}(x-f(y), y)-\sum_{i=1}^{q} \sharp \beta^{u}\left(f^{i}\right)(y) \alpha^{i}(x-f(y), y)\right)= \\
\sharp\left(\beta^{u}(x-f(y), y)-\sum_{i=1}^{q} \sharp \beta^{u}\left(f^{i}\right)(y) \frac{\partial}{\partial x^{i}}(x-f(y), y)=\phi_{*} \sharp \beta^{u}(x-f(y), y)\right.
\end{gathered}
$$

as expected also.

To achieve the result it remains to prove the formulae

$$
\left[\Phi \circ \alpha \circ \phi^{-1}, \Phi \circ \beta \circ \phi^{-1}\right]=\Phi \circ[\alpha, \beta] \circ \phi^{-1}
$$


for every pair $(\alpha, \beta)$ of sections. It suffices to verify this for sections in the basis. The two members of these equations are 0 if one of these sections is $\alpha^{i}$, so we have only to explore the case where $\alpha=\beta^{u}$ and $\beta=\beta^{v}$. Doing this we obtain

$$
\begin{gathered}
{\left[\Phi \circ \alpha \circ \phi^{-1}, \Phi \circ \beta \circ \phi^{-1}\right]=\left[\beta^{u}-\sum_{i=1}^{q} \sharp \beta^{u}\left(f^{i}\right) \alpha^{i}, \beta^{v}-\sum_{j=1}^{q} \sharp \beta^{v}\left(f^{j}\right) \alpha^{j}\right]} \\
=\left[\beta^{u}, \beta^{v}\right]-\sum_{i=1}^{q}\left[\sharp \beta^{u}, \sharp \beta^{v}\right]\left(f^{i}\right) \alpha^{i}=\Phi \circ[\alpha, \beta] \circ \phi^{-1}
\end{gathered}
$$

as expected.

In $[\mathrm{F}]$ the unicity, up to algebroid isomorphism, of the transversal algebroid to $F$ is proved (exactly as in the Poisson case in [W]) by a Moser's type - not so simpleargument. The preceding proposition simplifies the proof (in the algebroid case only). This proposition says also that, in the splitting theorem, we can impose that the coordinates $x$ vanish on any given transversal to the leaf of $m$.

4. Linearization. The splitting theorem reduces the local study of Lie algebroids to the case where the rank is 0 . This is the purpose of this section. From now on we suppose that our algebroid $A$ has a basis $M$ which is on open neighborhood of the origin in $\mathbb{R}^{s}$, with coordinates $x=\left(x_{1}, \ldots, x_{s}\right)$ and that we have a basis of sections $\left(\beta^{1}, \ldots, \beta^{r}\right)$. We write $\sharp \beta^{i}=\sum_{j=1}^{s} b^{i j} \frac{\partial}{\partial x^{j}}$, with $b^{i j}(0)=0$, and $\left[\beta^{i}, \beta^{j}\right]=\sum_{k=1}^{r} c_{k}^{i j} \beta^{k}$.

The linear part of $A$ at the origin is the Lie algebroid obtained from $A$ where we replace $b^{i j}$ by $\sum_{k=1}^{s} \frac{\partial b^{i j}(0)}{\partial x^{k}} x^{k}$ and $c_{k}^{i j}$ by $c_{k}^{i j}(0)$. In fact this linear part has an intrinsic definition (see $[\mathrm{F}]$ ). Note that this linear part is what is called an "action algebroid" ([W2]): the $\beta^{i}$ form the basis of a finite dimensional Lie algebra $G$ (with $\left[\beta^{i}, \beta^{j}\right]_{G}=\sum_{k=1}^{r} c_{k}^{i j}(0) \beta^{k}$ ) and the $\sharp \beta^{i}$ give an infinitesimal action of $G$ on $M$. Moreover, in that precise case, this action is linear.

We say that $A$ is linearizable if there is a change of coordinates and a change of the basis of sections which reduce $A$ to its linear part. In [W2] they are some basic insights concerning this notion in the case where $G$ is semi-simple. We will give these results hereafter.

THEOREM 6. With the notations above, if $G$ is semi-simple we can formally linearize A, i.e. we can linearize up to flat terms.

Proof. We will proceed by induction: we will suppose that we have Taylor expansions

$$
c_{k}^{i j}=c_{k}^{i j}(0)+c_{k}^{i j(q)}+c_{k}^{i j(q+1)}+\cdots
$$

where $c_{k}^{i j(n)}$ are the terms of order $n$ in the coordinates $\left(x^{1}, \ldots, x^{s}\right)$ and $q$ is a fixed integer greater or equal to 1 .

We denote by $A^{(q)}$ the space of sections of $A$ of the form $\sum_{i=1}^{r} a_{i} \beta^{i}$ where the $a_{i}$ are homogeneous polynomial coefficients of degree $q$. There is an infinitesimal action of $G$ on $A^{(q)}$ given by

$$
\beta^{u} \cdot\left(\sum_{i=1}^{r} a_{i} \beta^{i}\right):=\sum_{i=1}^{r} a_{i}\left[\beta^{u}, \beta^{i}\right]_{G}+\sharp \beta^{u(1)}\left(a_{i}\right) \beta^{i},
$$

where $\sharp \beta^{u(1)}$ is the linear part of $\sharp \beta^{u}$ at the origin. 
The family $\left(c_{k}^{i j(q)}\right)_{i, j, k=1, \ldots, r}$ determines a Chevalley 2-cochain $c^{(q)}: G \times G \rightarrow A^{(q)}$ by the rule

$$
c^{(q)}\left(\beta^{i}, \beta^{j}\right)=\sum_{k=1}^{r} c_{k}^{i j(q)} \beta^{k} .
$$

It is elementary that the Jacobi identity for bracket of the algebroid gives that $c^{(q)}$ is a Chevalley cocycle. Now the semi-simplicity of $G([\mathrm{Ch}])$ gives that it is a coboundary. This means that there are homogeneous functions of degree $q, \theta_{j}^{i(q)}$, for $i, j=1, \ldots, r$, such that

$$
c_{k}^{i j(q)}=\sum_{u=1}^{r}\left(\theta_{u}^{j(q)} c_{k}^{i u}(0)-\theta_{u}^{i(q)} c_{k}^{j u}(0)-\theta_{k}^{u(q)} c_{u}^{i j}(0)\right)+\sharp \beta^{i(1)} \theta_{k}^{j(q)}-\sharp \beta^{j(1)} \theta_{k}^{i(q)} .
$$

Now we consider the new basis of sections given by $\tilde{\beta}^{i}=\beta^{i}-\sum_{j=1}^{r} \theta_{j}^{i(q)} \beta^{j}$. An elementary calculation shows that we have $\left[\tilde{\beta}^{i}, \tilde{\beta}^{j}\right]=\tilde{c}_{k}^{i j} \tilde{\beta}^{k}$ with

$$
\tilde{c}_{k}^{i j}=c_{k}^{i j}(0)+\tilde{c}_{k}^{i j(q+1)}+\cdots .
$$

So, in this new basis, we have erased the terms of degree $q$.

This shows how to eliminate step by step terms of degree $q$ via a polynomial change of the basis of type Id $+q$-order terms. This suffices to show that, up to a formal change of the basis of sections, we can suppose that the $c_{k}^{i j}$ are constants. This says that we are formally in the case of an action algebroid. Now it remains to linearize formally the action by classical technics $([\mathrm{H}])$.

Remarks. It is possible to improve this theorem. When we work in the $C^{\infty}$-case and when $G$ is a compact algebra, the techniques of J. Conn ([C2]) permit to reduce smoothly to the case of an action algebroid. Then the classical linearization theory for compact actions (using averaging process) permits to linearize smoothly. When we work in the analytic case, the same procedure (using then [C1]) permits to linearize analytically.

We note that the splitting theorem and this analytic linearization process can be thought of as a special case of the ones which appeared in $([\mathrm{Ce}])$. Also this last formal linearization theorem could be deduced from a result of A. Wade ([Wa]).

\section{References}

[CW] A. Cannas da Silva and A. Weinstein, Geometric Models for Noncommutative Algebras, Berkeley Mathematics Lectures, vol. 10, American Math. Soc., Providence, 1999.

[Ce] D. Cerveau, Distributions involutives singulières. Ann. Inst. Fourier 29 (3) (1979), 261294.

[Ch] C. Chevalley and S. Eilenberg, Cohomology theory of Lie groups and Lie algebras, Transactions AMS, 63 (1948).

[C1] J. F. Conn, Normal forms for analytic Poisson structures. Ann. of Math. 119 (2) (1984), $576-601$.

[C2] J. F. Conn, Normal forms for smooth Poisson structures. Ann. of Math. 121 (2) (1985), $565-593$. 
[F] R. L. Fernandes, Lie algebroids, holonomy and characteristic classes. Preprint DG/ 0007132 (jul 2000).

[H] R. Hermann, The formal linearization of a semisimple Lie algebra of vector fields about a singular point, Trans. Amer. Math. Soc. 130 (1968), 105-109.

[M] K. Mackenzie, Lie Groupoids and Lie Algebroids in Differential Geometry, London Math. Soc. Lecture Notes Series 124, Cambridge Univ. Press, 1987.

[Wa] A. Wade, Normalisation formelle de structures de Poisson, C. R. Acad. Sci. Paris Sér. I Math. 324 (1997), 531-536.

[W] A. Weinstein, The local structure of Poisson manifolds, J. Diff. Geometry 18 (1983), 523-557.

[W2] A. Weinstein, Linearization problems for Lie algebroids and Lie groupoids, Lett. Math. Phys. 52 (2000), 93-102. 